\title{
THE CURRENT LHC LIMITS FOR THE tBESS LAGRANGIAN
}

\author{
We investigate how the recent $L H C$ measurements limit the parameters of the hypothetical vector resonance triplet. The vector resonances \\ are assumed to represent bound states of hypothetical strongly-interacting new physics, a candidate of the extension of the Standard model. \\ Keywords: New vector resonances, LHC limits, tBESS effective Lagrangian.
}

\section{Introduction}

Even though the LHC experiments ATLAS and CMS achieved a spectacular success by discovering the $125 \mathrm{GeV}$ Higgs boson [1] it was more the beginning rather than the end of the struggle to uncover the character of physics beyond the Standard Model (SM). To this moment, it has not been settled down whether new physics takes the form of weakly coupled supersymmetry or strongly coupled composites; this can be translated into question whether the observed Higgs boson is a fundamental field or a bound state of hypothetical new strong interactions.

The search for answers to these questions requires the close interplay between theory and experiment. On the theory side, the formulation of possible extensions of the SM along with their predictions for suitable observables is needed. In this context, we have formulated the effective Lagrangian (called the tBESS model) $[2,3]$ that could provide the framework for the description and analysis of phenomena that could be observed at the LHC if new strong physics is what lies behind the SM extension. Our effective description includes an explicit presence of a new vector resonance triplet.

In our previous papers [2 - 4], the tBESS model was a subject of a thorough investigation in an attempt to find how its parameters are restricted by the existing measurements. In our most recent work [3], our model faced the confrontation with the latest LHC measurements performed by the ATLAS and CMS Collaborations. Among other things we calculated the model's predictions for the LHC cross sections of the vector resonance production processes considering various final states to which the produced resonance can decay. We found that in the case of no direct coupling of the vector resonance with fermions the
LHC data provide only weak or no restrictions for the parameters of our model through this observable. In particular, considering ten decay channels of the resonance, namely $W W, Z W, W H$. $Z H$, $j j_{\text {neutral }}, j j_{\text {charged }}, \ell l, \ell v, t t$ and $t b$, the $1 \mathrm{TeV}-1.5 \mathrm{TeV}$ resonances get excluded in the $W W$ and $Z W$ channels for certain values of the vector resonance gauge coupling $g^{\prime \prime}$. However, even this restriction can get weakened once the direct interactions of the vector resonance with the fermion sector are introduced. None of the other reviewed decay channels excludes our model within the considered range of the values of $g^{\prime \prime}$.

We would like to extend the analysis of the resonance production cross section limits to the case when the direct interactions of the vector resonance with fermions are turned on. We believe that the presence of the direct interaction of the resonance to heavy fermions represents a more realistic case of new strong physics. It also offers a richer phenomenology to be investigated. The goal of this paper is to prepare and test the means necessary for performing such an analysis. It includes an upgrade of the means that were used in [3]. We will replace the parton distribution function set CTEQ6L1 used in [3] for the set CT10 which is more suitable for the LHC processes. In [3], we have used the vector boson luminosity functions calculated for the $14 \mathrm{TeV}$ collision energy. These will be replaced with the $13 \mathrm{TeV}$ functions so that they correspond to the actual collision energy at which the data were taken.

In the next section we will briefly introduce the tBESS model. In Section 3 the procedure for the calculation of the production cross section will be presented. Section 4 shows the results obtained for the $e^{+} e^{-}+\mu^{+} \mu^{-}$final state. The comparison of the results of the upgraded calculations with the results of [3] is performed. Finally, Section 5 presents conclusions of the paper.

\footnotetext{
* 1,2 Mikulas Gintner, ${ }^{2,3}$ Josef Juran

${ }^{1}$ Physics Department, University of Zilina, Slovakia

${ }^{2}$ Institute of Experimental and Applied Physics, Czech Technical University in Prague, Czech Republic

${ }^{3}$ Institute of Physics, Silesian University in Opava, Czech Republic

E-mail: gintner@fyzika.uniza.sk
} 


\section{The effective tBESS Lagrangian}

The effective tBESS Lagrangian [2,3] serves as the effective description of a hypothetical strongly interacting extension of the SM where the principal manifestation of this scenario at the LHC energies would be the existence of a vector resonance triplet as a bound state of new strong interactions. The Lagrangian is built to respect the global $S U(2)_{L} \times S U(2)_{R} \times U(1)_{B-L} \times S U(2)_{H L S}$ symmetry of which the $S U(2)_{L} \times U(1)_{Y} \times S U(2)_{H L S}$ subgroup is also a local symmetry. The $S U(2)_{H L S}$ symmetry is an auxiliary gauge symmetry invoked to accommodate the $S U(2)$ triplet of new vector resonances. Each of the gauge groups is accompanied by its gauge coupling: $g, g^{\prime}, g^{\prime \prime}$ respectively. Beside the scalar singlet representing the $125 \mathrm{GeV}$ Higgs boson and the hypothetical vector triplet, the effective Lagrangian is built out of the SM fields only.

The way the description of the vector resonance is introduced implies the mixing between the resonance and electroweak gauge boson fields. The mixing induces the (indirect) couplings between the vector resonance and fermions that are proportional to $1 / g^{\prime \prime}$. The considered symmetry also admits the introduction of the direct interactions of the vector resonance with fermions. The existing experimental limits inspired the tBESS specific assumption that only the third quark doublet couples directly to the vector resonance. This chiral coupling is parameterized by the $b_{L, R}$ parameters. In addition, the vector resonance coupling to the right bottom quark can be made weaker than the right top coupling. The ratio is parameterized by $p \in\langle 0 ; 1\rangle$. Finally, the symmetry admits a further modifications of the electroweak gauge boson couplings to fermions parameterized by $\lambda_{L, R}$.

The deviations from the SMinteractions of the $S U(2)_{L} \times U(1)_{Y}$ gauge boson with the Higgs boson is parametrized by $a_{V}$. The analogical coupling of the vector resonance to the Higgs is parametrized by $a_{\rho}$.

The masses of the charged and neutral vector resonances in the model are virtually degenerated. The leading order formula for the mass reads

$M_{\rho}=\frac{v}{2} \sqrt{\alpha} g^{\prime \prime}$

where $\alpha$ is a dimensionless free parameter in the tBESS Lagrangian and $v$ is the electroweak symmetry breaking scale. Usually, $\alpha$ is traded off for $M_{\rho}$ so that the latter can serve as one of the free parameters of the model. Our previous studies of the low-energy [2], Higgs-related [3] and unitarity limits [2, 3] suggest that we should consider $10 \leq g^{\prime \prime} \leq 20$ and $1 \mathrm{TeV} \leq M_{\rho} \leq 3 \mathrm{TeV}$. Furthemore, $a_{V}=1$ and $a_{\rho}=0$ are quite close to one of the experimentally preferred point of the parameter space. Therefore these will be the values we use throughout this paper. Note though that the dependence of the results presented below on $a_{V}$ and $a_{\rho}$ will be negligible. These parameters influence significantly only decay channels of the vector resonance with very small branching ratios.

In this paper, we will consider no direct interactions of the vector resonance with fermions $\left(b_{L, R}=p=\lambda_{L, R}=0\right)$. Then, the total decay width of the resonance can be approximated by

$\Gamma_{\rho}=\frac{1}{48 \pi v^{4}} \frac{M_{\rho}^{5}}{g^{\prime \prime 2}}$.

In Fig. 1 we depict how the vector resonance width in this approximation depends on the resonance mass and $g^{\prime \prime}$.

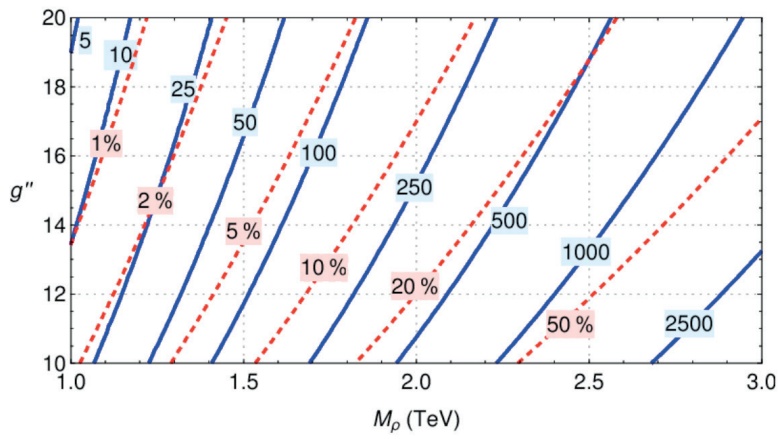

Fig. 1 The solid lines represent the contours of the total decay width of the vector resonance (labeled in $\mathrm{GeV}$ ) in the $g^{\prime \prime}-M \rho$ parameter space; the dashed lines represent the width-to-mass ratio of the vector resonance (labeled in percents); no direct interactions of the resonance to fermions is assumed

\section{The cross section calculations}

We are interested in the LHC cross sections of the two-particle final state processes that would proceed via the vector resonance production. We calculate the cross sections $\sigma(p p \rightarrow a+b+X)$ in the Narrow Width Approximation (NWA), i.e. we approximate the cross sections by the product of the on-shell production cross section of the resonance, $\sigma_{\text {prod }}$, and the branching ratio for the vector resonance decay channel under consideration

$\sigma(p p \rightarrow a b+X) \approx \sigma^{N W A}=\sigma_{p r o d}(p p \rightarrow \rho+X) \times$ $\times B R(\rho \rightarrow a b)$

As the name suggests the NWA works when $\Gamma_{\rho} \ll M_{\rho}$. It also ignores the signal-background interference effects. The influence of these effects on the precision of the approximation has been investigated in [5].

In general, the production cross section of a resonance can be expressed as

$\sigma_{\text {prod }}(p p \rightarrow \rho+X)=\left.\sum_{i \leq j \in p} 16 \pi^{2} K_{i j} \frac{\Gamma_{\rho \rightarrow i j}}{M_{\rho}} \frac{d L_{i j}}{d \hat{s}}\right|_{\hat{s}=M_{\rho}^{2}}$

where $i, j$ run through all partons of the colliding protons and $\Gamma_{\rho \rightarrow i j}$ is the partial decay width of the resonance to the partons. 
Furthermore, $d L_{i j} / d \hat{s}$ is a parton-parton luminosity of the colliding partons, and

$$
K_{i j}=\frac{2 J+1}{\left(2 S_{i}+1\right)\left(2 S_{j}+1\right)} \frac{C}{C_{i} C_{j}}
$$

where $J$ is a spin of the resonance, $C$ is its color factor, and $S_{i}, S_{j}$ and $C_{i}, C_{j}$ are the spins and colors of the initial partons, respectively. Note that a model dependence enters the production cross section (4) virtually ${ }^{1}$ only via the partial decay width $\Gamma_{\rho \rightarrow i j}$.

Two dominant production mechanisms for the triplet of our vector resonances are the Drell-Yan (DY) and the vector boson fusion (VBF) processes. We will consider them both in our analysis. The VBF production will be calculated in the Effective $W$ Approximation (EWA) [6]. For the sake of simplicity, in our calculations of the production cross sections the proton contents is reduced down to the up and down quarks ${ }^{2}$. Thus, the DY partons include $\{u, \bar{u}, d, \bar{d}\}$ and the VBF partons are $\left\{W^{+}, W^{-}, Z\right\}$. When the vector resonance $(J=1, C=1)$ is produced, the factor $K_{i j}$ in the formula (4) assumes the value $K^{D Y}=1 / 12$ in the case of the DY production; $K^{V B F}=1 / 3$ for the VBF process. Any of the three charged states of the vector resonance $\rho$ can be produced: $\rho^{ \pm}$can be created in the $u \bar{d} / \bar{u} d(\mathrm{DY})$ and $W^{ \pm} Z(\mathrm{VBF})$ collisions, while the neutral resonance $\rho^{0}$ can be produced in $u \bar{u}, d \bar{d}(\mathrm{DY})$ and $W^{+} W^{-}(\mathrm{VBF})$ collisions. If the b-quark contents of the proton is ignored the production cross section is not sensitive to the parameters $b_{L, R}, p, \lambda_{L, R}$.

The parton-parton luminosity appearing in (4) is defined as

$\frac{d L_{i j}}{d \hat{s}}=\frac{1}{S} \int_{\tau}^{1} \frac{d x}{x} \frac{1}{1+\delta_{i j}}\left[f_{i}(x, \hat{s}) f_{j}(\tau / x, \hat{s})+i \leftrightarrow j\right]$

where $s$ and $\hat{s}$ are the squared center of mass energies of the colliding protons and partons, respectively, $\tau \equiv \hat{s} / s$, and $f_{i}$ is a parton distribution function of the $i^{\text {th }}$ parton with the momentum fraction $x$ of its proton's momentum. In the DY case, $f_{i}$ 's are the parton distribution functions (PDF) of quarks and gluons in the proton. When the EWA is applied to the VBF case, the $W$ and $Z$ bosons are also treated as partons in the proton. In this approximation, their PDF's are calculated as the convolutions of the quark PDF's with the probability that a given quark would emit $W$ or $Z$ boson. Then the VBF luminosity can be expressed as

$$
\begin{aligned}
\frac{d L_{\left.V_{m} V_{[}[p]\right]}}{d \tau}= & \sum_{i \leq j} \frac{1}{1+\delta_{i j}} \int_{\tau}^{1} \frac{d x_{1}}{x_{1}} \int_{\tau / x_{1}}^{1} \frac{d x_{2}}{x_{2}}\left[f_{i}\left(x_{1}, q^{2}\right) f_{j}\left(x_{2}, q^{2}\right) \times\right. \\
& \left.\frac{d L_{V_{m} V_{n}[q i q]}}{d \hat{\tau}}+i \leftrightarrow j\right]
\end{aligned}
$$

\footnotetext{
${ }^{1}$ In principle, the parton-parton luminosity is also sensitive to new physics via modifications of the SM couplings and the parton distribution functions. Nevertheless, we expect these effects to be negligible and ignore them in our analysis.

${ }^{2}$ We do not expect that this approximation would significantly influence conclusions of this study since we assume no direct interactions of the vector resonance to fermions. The investigation of the more general case with the direct interactions present is in progress.
}

where $\hat{\tau}=\tau /\left(x_{1} x_{2}\right)$, and $d L_{V_{m} V_{n}\left[q_{1} q_{2}\right]} / d \hat{\tau}$ is the luminosity for two vector bosons $V_{m}$ and $V_{n}$ emitted from $i^{\text {th }}$ and $j^{\text {th }}$ quarks, respectively,

$\frac{d L_{V_{m} V_{n}\left[q_{1} q_{2}\right]}}{d \hat{\tau}}=\int_{\hat{\tau}}^{1} \frac{d x}{x} f_{V_{m}\left[q_{i}\right]}(x) f_{V_{n}\left[q_{j}\right]}(\hat{\tau} / x)$

where $f_{V_{m}\left[q_{i}\right]}$ 's represent the gauge boson contents of quarks in the same way the PDF's represent the quark/gluon contents of protons. Contrary to the PDF's case though, we know how to calculate $f_{V_{V_{m}}\left[q_{i}\right]}$ 's from the first principles. Finally, the scale $q^{2}$ in (7) will be set to $M_{W}^{2}$.

The EWA's PDF's are derived under the assumptions that the gauge bosons are emitted on-shell and in small angles to their parental quarks. Also, if the gauge bosons fuse to a heavy resonance their masses should be negligibly small compare to the resonance mass. Moreover, in this approximation, the transverse and longitudinal polarizations of the emitted gauge bosons are considered as separate modes and, in some cases, one of them becomes negligible to the other. For example, in the SM the VBF production is dominated by the transverse mode due to the exact mutual cancellation of the ill-behaved longitudinal gauge boson and Higgs amplitudes. On the other hand, new physics contributions are expected to tip this balance and let dominate the longitudinal mode as the collision energy grows. Since we deal with new physics in our analysis, we restrict our calculations to the use of the longitudinal mode only.

The luminosity for two longitudinal vector bosons $V_{m}$ and $V_{n}$ emitted from $i^{\text {th }}$ and $j^{\text {th }}$ quarks reads

$$
\begin{aligned}
& \frac{d L_{V_{m}^{L} V_{[}^{L}\left[q_{i q j}\right]}}{d \hat{\tau}}=\frac{v_{m[i]}^{2}+a_{m[i]}^{2}}{4 \pi^{2}} \frac{v_{n[i]}^{2}+a_{n[i]}^{2}}{4 \pi^{2}} \frac{1}{\hat{\tau}} \times \\
& {[(1+\hat{\tau}) \log (1 / \tau)-2(1-\hat{\tau})]}
\end{aligned}
$$

where $v_{m[i]}$ and $a_{m[i]}$ are the vector and axial couplings of the gauge boson $V_{m}$ to the quark current $q_{i}$. In particular,

$v_{W\left[q_{i}\right]}=-a_{W\left[q_{i}\right]}=\frac{q}{2 \sqrt{2}}$

for any $q_{i}$, and

$v_{Z[u]}=\frac{g}{4 c_{W}}\left(1-\frac{8}{3} s_{W}^{2}\right), a_{Z[u]}=\frac{g}{4 c_{W}}$

$v_{z[d]}=-\frac{g}{4 c_{W}}\left(1-\frac{4}{3} s_{W}^{2}\right), a_{z[d]}=-\frac{g}{4 c_{W}}$

where $s_{W}=\sin \theta_{W}$ and $c_{W}=\cos \theta_{W}$.

For the numerical evaluation of the parton-parton luminosity $d L_{i j} / d \hat{s}$ we used the Mathematica [8] package Mane Parse [9] with the PDF set CT10 from the LHAPDF 6 library at HepForge repository [7]. The obtained parton-parton luminosities for both production mechanisms of the new vector resonance at the LHC $(\sqrt{s}=13 \mathrm{TeV})$ are depicted in Fig. 2 . 


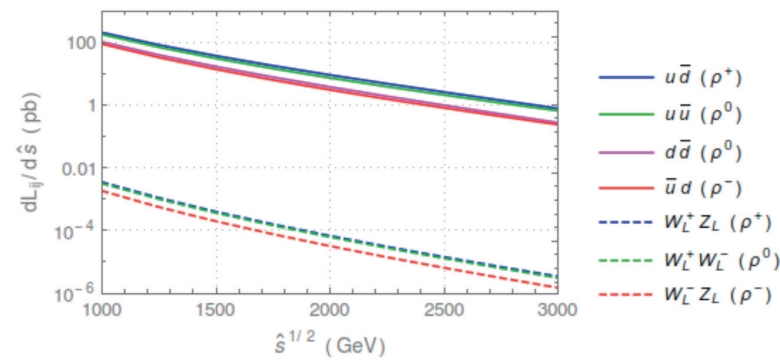

Fig. 2 The parton-parton luminosities for the DY (solid lines) and longitudinal VBF (dashed lines) production in the proton-proton collisions at $\sqrt{\mathrm{s}}=13 \mathrm{TeV}$; the CT10 set [7] of parton distribution functions was used

As we can see the DY luminosities dominate over VBF ones by about $4-5$ orders of magnitude. However, since the partial decay widths $\Gamma_{\rho \rightarrow W Z}$ and $\Gamma_{\rho \rightarrow W W}$ grow with the fifth power of the resonance mass their contributions can significantly increase the VBF production cross sections when heavier resonances are considered. This can be observed in the graph of the production cross sections shown in Fig. 3 for $g^{\prime \prime}=15$. Note that in the mass region above $2 \mathrm{TeV}$ the $\Gamma_{\rho} / M_{\rho}$ ratio exceeds $10 \%$ which increases tension between reality and the assumptions for the NWA.

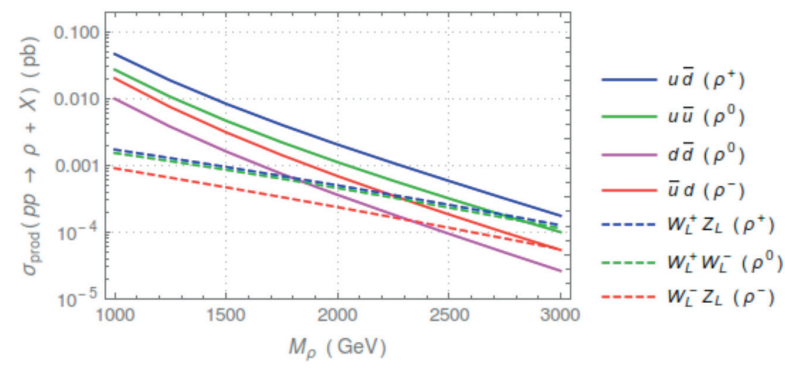

Fig. 3 The production cross sections of the new vector resonance via various initial states (see the legend) in proton-proton collisions at $\sqrt{s}=13 \mathrm{TeV}$ as a functions of $M_{\rho}$. The solid lines represent the $D Y$ processes, the dashed lines are for VBF processes. The shown curves were calculated at $g^{\prime \prime}=15$

\section{Results for the dilepton channel}

Once the production cross sections have been evaluated, we are a single step from finalizing the predictions of the LHC cross sections of the processes under investigation. As we can see in (3) the final step involves the multiplication of the production cross section with the appropriate branching ratio. The branching ratios of the various decay channels of the resonance are those principal quantities through which the final cross section receives its sensitivity to the model under consideration. Thus, in this stage, we are in the position of investigating the cross section dependence on various model parameters. In addition, these predictions can be confronted with the experimental measurements provided by the LHC experiments. Consequently, various experimental restrictions on the model parameters can be derived.

The thorough analysis suggested at the end of the previous paragraph is not within the scope of this paper. Here, we will demonstrate only how much the results obtained by the upgraded computational approach influences the results obtained in our previous paper [3]. For that sake, we have calculated the cross section of the process with dileptons in the final state: $p p \rightarrow \ell \bar{\ell}+X$, where $\ell=e, \mu$. While this is not the dominant decay channel it is not very sensitive to the fermion couplings of the vector resonance and thus the obtained prediction is quite universal in this sense. The low sensitivity and the low cross section infers from the fact that light fermions do not couple directly to the vector resonances in our model. The interaction occurs only via the vector-boson mixing and is proportional to $1 / g^{\prime \prime}$. From the experimental point of view, the dielectron and dimuon final states have the advantage of a relatively clean signal. The branching ratios of the combined dielectron and dimuon final states for various combinations of the values of $g^{\prime \prime}$ and $M_{\rho}$ are shown in Table 1.

Using the dilepton branching ratios we calculate the cross section $\sigma\left(p p \rightarrow e^{+} e^{-}+\mu^{+} \mu^{-}+X\right)$ at $\sqrt{s}=13 \mathrm{TeV}$. The result as a function of $M_{\rho}$ and for three different values of $g^{\prime \prime}$ is shown in Fig. 4. In addition, the graph of predicted cross section is superimposed with the ATLAS Collaboration curve showing the expected upper $95 \%$ C.L. limit on the cross section times branching ratio [10]. The current experimental data do not contradict the ATLAS expectation curve [10] so we take it as the boundary of the sensitivity of the existing measurements in this channel. We can see that the predictions of our model lie well below this boundary. Thus none of the considered resonance masses are ruled out by the LHC measurement in this channel. This confirms our findings in [3].

The branching ratios of the combined dielectron and dimuon final states for various combinations of the values of $g^{\prime \prime}$ and $M_{\rho}$

Table 1

\begin{tabular}{|c|c|c|c|c|c|}
\hline \multicolumn{6}{|c|}{$B R\left(\rho^{0} \rightarrow e^{+} e^{-}+\mu^{+} \mu^{-}\right) \times 10^{3}$} \\
\hline$M_{\rho}(\mathrm{GeV})$ & 1000 & 1500 & 2000 & 2500 & 3000 \\
\hline$g^{\prime \prime}=10$ & 1.224 & 0.2529 & 0.08127 & 0.03353 & 0.01623 \\
\hline$g^{\prime \prime}=15$ & 1.223 & 0.2528 & 0.08125 & 0.03352 & 0.01623 \\
\hline$g^{\prime \prime}=20$ & 1.223 & 0.2528 & 0.08124 & 0.03352 & 0.01623 \\
\hline
\end{tabular}




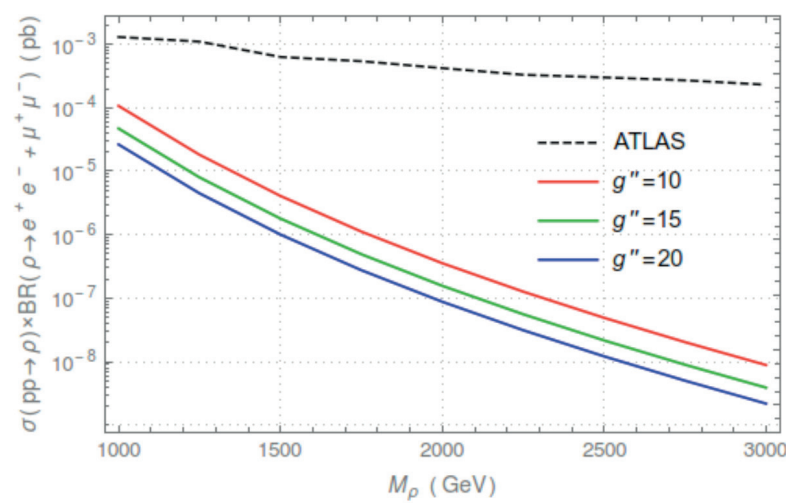

Fig. 4 The LHC production cross section times the branching ratio as a function of $M_{\rho}$ for the $e^{+} e^{-}+\mu^{+} \mu^{-}$final state at $\sqrt{s}=13 \mathrm{TeV}$ calculated for three different values of the parameter $g^{\prime \prime}$ (solid lines); the dashed line shows the expected upper $95 \%$ C.L. limit obtained by the ATLAS Collaboration

The main goal of this work was to develop and test the upgraded version of the cross section calculations so that it can be used in a more complex analysis of the current limits for the tBESS model parameters. When our upgraded prediction of $\sigma\left(p p \rightarrow e^{+} e^{-}+\mu^{+} \mu^{-}+X\right)$ is compared with our previous calculations in [3] we find a difference at a level of severa percents. The difference grows with the mass of the resonance. In particular, the upgraded calculation exceeds the previous prediction by about $0.5 \%$ when $M_{\rho}=1 \mathrm{TeV}$ and falls below it by about $5 \%$ when $M_{\rho}=2 \mathrm{TeV}$. We believe that our upgrade have had the most significant impact on the calculations of the VBF production. Since the contribution of the VBF production grows with $M_{\rho}$ (see Fig. 3) this could explain the observed tendency in the discrepancy behaviour.

\section{Conclusions}

We have calculated the vector resonance production cross section for the $e^{+} e^{-}+\mu^{+} \mu^{-}$final state as a function of the vector resonance mass and for various values of $g^{\prime \prime}$ within the effective description of new physics provided by the tBESS model. The predicted cross section is significantly below the sensitivity of the latest ATLAS measurements in this channel. Thus, the data provide no restrictions for the model there. This is in the agreement with our previous findings based on more rudimentary calculations in [3]. The upgraded calculations in this paper differ from the results in [3] from 0.5 to $5 \%$ when $M_{\rho}$ ranges from $1 \mathrm{TeV}$ to $2 \mathrm{TeV}$. The upgrade of the calculations included the use of the CT10 set for the PDF's of protons and the use of the vector boson luminosity functions calculated precisely at the collision energy at which the used data had been collected.

This calculation was a part of preparing steps for more thorough analysis that would confront the tBESS model with the current LHC data in all measured channels: $W W, Z W, W H, Z H$, $j j_{\text {neutral }}, j j_{\text {charged }}, \ell l, \ell v, t t$, and $t b$. Beside analyzing all additional channels with a more restrictive potential expected from some, we will also turn on the direct interactions of the vector resonance with heavy fermions. It can provide the explanation why such a new vector resonance avoids its discovery at the LHC.

\section{Acknowledgments}

We would like to thank Karol Kovarik for useful discussions. The work of M.G. and J.J. was supported by the Grants LTT17018 and LG15052 of the Ministry of Education, Youth and Sports of the Czech Republic. M.G. was supported by the Slovak CERN Fund. J.J. was supported by the National Scholarship Programme of the Slovak Republic.

\section{References}

[1] AAD, G., et al. (ATLAS Collaboration): Observation of a New Boson at a Mass of $125 \mathrm{GeV}$ with the CMS Experiment at the LHC. Physics Letters B, 716(1), 30-61, 2012; CHATRCHYAN, S., et al. (CMS Collaboration), ibid. 30, 2012.

[2] GINTNER, M., JURAN, J.: The Vector Resonance Triplet with the Direct Coupling to the Third Quark Generation. The European Physical Journal C, 73, 2577, 2013.

[3] GINTNER, M., JURAN, J.: The Limits on the Strong Higgs Sector Parameters in the Presence of New Vector Resonances. The European Physical Journal C, 76, 651, 2016; erratum: The European Physical Journal C, 77, 6, 2017.

[4] GINTNER, M., JURAN, J, MELO, I.: Top-BESS Model and its Phenomenology. Physical Review D, 84, 035013, 2011.

[5] PAPPADOPUlO, D., THAMM, A., TORRE, R., WULZER, A.: Heavy Vector Triplets: Bridging Theory and Data. Journal of High Energy Physics, 1409, 060, 2014.

[6] DAWSON, S.: The Effective W Approximation. Nuclear Physics B, 249(1), 42-60, 1985.

[7] BUCKLEY, A., et al.: LHAPDF6: Parton Density Access in the LHC Precision Era [online]. The European Physical Journal C, 75, 132, 2015. Available: http://hapdf.hepforge.org/pdfsets.

[8] WOLFRAM RESEARCH, Inc.: Mathematica, Version 10.4. Champaign, IL., 2016. 


\section{COMMNICOIIONS}

[9] CLARK, D. B., GODAT, E., OLNESS, F. I.: ManeParse: A Mathematica Reader for Parton Distribution Functions [online]. Available: https://ncteq.hepforge.org/mma/index.html.

[10] ATLAS COLLABORATION: Search for New High-Mass Resonances in the Dilepton Final State Using Proton-Proton Collisions at $\sqrt{\mathrm{s}}=13 \mathrm{TeV}$ with the ATLAS detector. ATLAS-CONF-2016-045, Switzerland, 2016. 